International Journal of Bifurcation and Chaos, Vol. 24, No. 4 (2014) 1450042 (12 pages)

(C) World Scientific Publishing Company

DOI: $10.1142 / \mathrm{S} 0218127414500424$

\title{
Bifurcation Analysis of a Generic Reaction-Diffusion Turing Model
}

\author{
Ping Liu* \\ Y.Y.Tseng Functional Analysis Research Center \\ and School of Mathematical Sciences, \\ Harbin Normal University, Harbin, \\ Heilongjiang 150025, P. R. China \\ liuping506@gmail.com \\ Junping Shi ${ }^{\dagger}$ \\ Department of Mathematics, \\ College of William and Mary, Williamsburg, \\ Virginia 23187-8795, USA \\ jxshix@wm.edu \\ Rui Wang $\ddagger$ and Yuwen Wang§ \\ Y.Y.Tseng Functional Analysis Research Center \\ and School of Mathematical Sciences, \\ Harbin Normal University, Harbin, \\ Heilongjiang 150025, P. R. China \\ ¥diyishijian01@163.com \\ §wangyuwen1950@gmail.com
}

Received June 15, 2012; Revised December 19, 2012

\begin{abstract}
A generic Turing type reaction-diffusion system derived from the Taylor expansion near a constant equilibrium is analyzed. The existence of Hopf bifurcations and steady state bifurcations is obtained. The bifurcation direction and the stability of the bifurcating periodic obits are calculated. Numerical simulations are included to show the rich spatiotemporal dynamics.
\end{abstract}

Keywords: Turing reaction-diffusion model; Hopf bifurcation; steady state bifurcation.

\section{Introduction}

In 1952, Alan Turing published a seminal paper "The chemical basis of morphogenesis" [Turing, 1952]. His intriguing ideas influenced the thinking of theoretical biologists and scientists from many fields, successfully developed on the theoretical backgrounds [Murray, 1982; Ni \& Tang, 2005; Satnoianu et al., 2000; Segel \& Jackson, 1972; Szili \&
Tóth, 1997]. The Turing mechanism has been successfully adopted to explain pattern formation in diverse biological examples, including regeneration of hydra [Gierer \& Meinhardt, 1972; Meinhardt, 1982], coat of mammals [Murray, 1981, 2002/03], fish skin [Asai et al., 1999; Kondo \& Asai, 1995; Painter et al., 1999], sea shells [Meinhardt, 1995], feather [Jung et al., 1998] and so on. In chemistry,

\footnotetext{
*Partially supported by NSFC grant 11101110 and NCET grant 1251-NCET-002.

${ }^{\dagger}$ Partially supported by NSF grant DMS-1022648 and DMS-1313243.

§Partially supported by NSFC Grant 11071051.
} 
the experimental observation of a chemical Turing pattern was achieved on operating the chlorideiodide-malonic acid (CIMA) reaction in an open spatial reactor in 1990 [De Kepper et al., 1991; Ouyang \& Swinney, 1991; Rudovics et al., 1999]. The experiment on the CIMA reaction has revealed the existence of stationary spatially periodic concentration patterns. For a theoretical approach, there is a variety of Turing models with different reaction kinetics and their own specific characteristics, for instance, the well-known models like the Brusselator, Gray-Scott model and LengyelEpstein model [Gray \& Scott, 1990; Nicolis \& Prigogine, 1977].

In this paper, we discuss a reaction-diffusion system which is derived from the Taylor expansion of a generic Turing reaction-diffusion model around a constant equilibrium point (see [Barrio et al., 1999]):

$$
\left\{\begin{array}{r}
u_{t}=D \delta \Delta u+\alpha u\left(1-r_{1} v^{2}\right)+v\left(1-r_{2} u\right), \\
x \in \Omega, \quad t>0, \\
v_{t}=\delta \Delta v+v\left(\beta+\alpha r_{1} u v\right)+u\left(-\alpha+r_{2} v\right), \\
x \in \Omega, \quad t>0, \\
u(x, 0)=u_{0}(x), \quad v(x, 0)=v_{0}(x), \quad x \in \Omega, \\
\partial_{\nu} u=\partial_{\nu} v=0, \quad x \in \partial \Omega, \quad t>0,
\end{array}\right.
$$

in the spatial domain $\Omega=(0, l \pi), l \in \mathbb{R}^{+}$with no-flux boundary conditions. Here $D$ is the ratio of the two diffusion coefficients, $\delta$ is the diffusion coefficient of the second species; and kinetic parameters are $r_{1}, r_{2}, \alpha, \beta$, where $r_{1}, r_{2}$ are the cubic and quadratic coefficients of the Taylor polynomial, respectively. This model is dubbed as BarrioVarea-Aragon-Maini (BVAM) model [Leppänen et al., 2004]. Note that this model is a phenomenological one, and it is not based on any realistic experimental chemical reaction. However the BVAM model is the simplest system containing both quadratic and cubic nonlinear terms, and one can adjust the relative strength of the quadratic and cubic nonlinearities to see the effect on the pattern formation [Leppänen et al., 2004]. Hence a better understanding of the dynamics of the BVAM model can lead to the advancement of the studies of spatiotemporal pattern formation in general.

Two-dimensional Turing patterns of (1) in a square domain were analyzed and simulated in
[Barrio et al., 1999], and the patterns in a twodimensional disk were considered in [Aragón et al., 2002] and [Barrio et al., 2002]. Three-dimensional patterns have been considered in [Leppänen et al., 2002] and [Leppänen et al., 2004], and traveling wave solution was analyzed in [Varea et al., 2007]. Note that in all these works except [Varea et al., 2007], only steady state solutions (patterns) have been considered, and the studies are based on linearized analysis and numerical simulation.

In this paper, we consider both the timeperiodic patterns (periodic orbits) and stationary patterns (steady state solutions) of (1). We use bifurcation theory to rigorously prove the existence of Hopf bifurcations and steady state bifurcations of (1) for the whole parameter region satisfying $D>0, \delta>0,-1<\beta<0,0<\alpha<1$ and $r_{1}, r_{2}>0$, which is chosen this way so the Turing instability condition is satisfied [Barrio et al., 1999]. We show that nonconstant time-periodic patterns and stationary patterns can emerge from the unique positive constant steady state by varying a parameter $\alpha$.

Our analysis follows a general framework given in [Yi et al., 2009] for a diffusive RosenzeigMacArthur predator-prey systems, and a similar approach has also been taken in [Han \& Bao, 2009; Jin et al., 2013; Liu et al., 2010; Liu et al., 2013; Wang et al., 2011; Xu \& Wei, 2012; Yi et al., 2010] for various reaction-diffusion models. Because of the simplicity of the reaction terms in the BVAM model, a rather complete classification of patterns generated in a one-dimensional domain is obtained here (see Sec. 4), which is not possible for most previous works as their nonlinearities are more complicated. The time-periodic patterns and stationary spatial patterns are both among the six possible spatiotemporal patterns of reaction-diffusion systems first given by Turing [1952] in his seminal work 60 years ago. The impact of delay on the reactiondiffusion systems has also been considered recently [Chen et al., 2012, 2013; Seirin Lee et al., 2010; Su et al., 2009; Wijeratne et al., 2009; Yan \& Li, 2008, 2009].

In Sec. 2, Hopf bifurcation analysis with parameter $\alpha$ is conducted, and in Sec. 3, the steady state solution bifurcations are proved. Some numerical simulations are given in Sec. 4 to illustrate our results. In this paper, we denote the set of all the positive integers by $\mathbb{N}$, and $\mathbb{N}_{0}=\mathbb{N} \cup\{0\}$. 


\section{Hopf Bifurcations}

In this section, we consider the Hopf bifurcations for the generic Turing reaction-diffusion model (1). We consider one-dimensional space domain $\Omega=(0, l \pi)$, for which the structure of the eigenvalues is clear. So Eq. (1) is now in the following form

$$
\left\{\begin{array}{r}
u_{t}=D \delta u_{x x}+\alpha u\left(1-r_{1} v^{2}\right)+v\left(1-r_{2} u\right), \\
x \in(0, l \pi), \quad t>0, \\
v_{t}=\delta v_{x x}+v\left(\beta+\alpha r_{1} u v\right)+u\left(-\alpha+r_{2} v\right), \\
x \in(0, l \pi), t>0, \\
u_{x}(0, t)=u_{x}(l \pi, t)=v_{x}(0, t)=v_{x}(l \pi, t)=0, \\
t>0, \\
u(x, 0)=u_{0}(x), \quad v(x, 0)=v_{0}(x), \quad x \in(0, l \pi) .
\end{array}\right.
$$

First, we consider the corresponding kinetic equation of $(1)$, that is

$$
\begin{cases}u^{\prime}=\alpha u\left(1-r_{1} v^{2}\right)+v\left(1-r_{2} u\right), & t>0, \\ v^{\prime}=v\left(\beta+\alpha r_{1} u v\right)+u\left(-\alpha+r_{2} v\right), & t>0, \\ u(0)=u_{0}, \quad v(0)=v_{0} . & \end{cases}
$$

One can verify that $(0,0)$ is the unique equilibrium point of (3). In the following, we choose fixed parameters $-1<\beta<0, r_{1}>0, r_{2}>0$, and use $\alpha$ as the main bifurcation parameter. The linearized operator of the ODE system in (3) evaluated at $(0,0)$ is

$$
L_{0}(\alpha)=\left(\begin{array}{rr}
\alpha & 1 \\
-\alpha & \beta
\end{array}\right) .
$$

The characteristic equation of $L_{0}(\alpha)$ is

$$
\mu^{2}-\mu T(\alpha)+D(\alpha)=0,
$$

where $T(\alpha)=\alpha+\beta, D(\alpha)=\alpha(\beta+1)$. The eigenvalues $\mu(\alpha)$ of $L_{0}(\alpha)$ are given by

$$
\mu(\alpha)=\frac{\alpha+\beta \pm \sqrt{(\alpha-\beta)^{2}-4}}{2} .
$$

A Hopf bifurcation value $\alpha$ for (3) satisfies the following condition:

$$
T(\alpha)=0, \quad D(\alpha)>0, \quad \text { and } \quad T^{\prime}(\alpha) \neq 0 .
$$

Clearly we always have $D(\alpha)>0$ for $0<\alpha<1$ and $-1<\beta<0$, and $T(\alpha)=0$ implies $\alpha=-\beta$. Finally, $T^{\prime}(\alpha)=1$. Then $\alpha=-\beta$ is the only
Hopf bifurcation point for (3). When $0<\alpha<-\beta$, the unique equilibrium point $(0,0)$ of $(3)$ is always locally asymptotically stable, and it is unstable for $-\beta<\alpha<1$.

Next we consider Hopf bifurcations from the constant equilibrium $(0,0)$ of the reaction-diffusion system (2). We choose the length parameter $l$ properly, and use $\alpha$ as the main bifurcation parameter. Define

$$
\begin{aligned}
X:=\left\{(u, v) \in H^{2}[(0, l \pi)] \times H^{2}[(0, l \pi)]:\right. \\
\left.u^{\prime}(0)=v^{\prime}(0)=u^{\prime}(l \pi)=v^{\prime}(l \pi)=0\right\} .
\end{aligned}
$$

The linearized operator of system (2) evaluated at $(0,0)$ is

$$
L(\alpha)=\left(\begin{array}{cc}
D \delta \frac{\partial^{2}}{\partial x^{2}}+\alpha & 1 \\
-\alpha & \delta \frac{\partial^{2}}{\partial x^{2}}+\beta
\end{array}\right) .
$$

It is well-known that the eigenvalue problem

$$
-\psi^{\prime \prime}=\mu \psi, \quad x \in(0, l \pi), \quad \psi^{\prime}(0)=\psi^{\prime}(l \pi)=0,
$$

has eigenvalues $\mu_{n}=\frac{n^{2}}{l^{2}}(n=0,1,2, \ldots)$, with corresponding eigenfunctions $\psi_{n}(x)=\cos \frac{n}{l} x$. Let

$$
\left(\begin{array}{l}
\phi \\
\varphi
\end{array}\right)=\sum_{n=0}^{\infty}\left(\begin{array}{l}
a_{n} \\
b_{n}
\end{array}\right) \cos \frac{n}{l} x
$$

be an eigenfunction for $L(\alpha)$ with eigenvalue $\mu(\alpha)$, that is, $L(\alpha)(\phi, \varphi)^{T}=\mu(\alpha)(\phi, \varphi)^{T}$. According to $\left[\mathrm{Yi}\right.$ et al., 2009], there exists $n \in \mathbb{N}_{0}$ such that $L_{n}(\alpha)\left(a_{n}, b_{n}\right)^{T}=\mu(\alpha)\left(a_{n}, b_{n}\right)^{T}$, where $L_{n}$ is defined as

$$
L_{n}(\alpha):=\left(\begin{array}{cc}
-D \delta \frac{n^{2}}{l^{2}}+\alpha & 1 \\
-\alpha & -\delta \frac{n^{2}}{l^{2}}+\beta
\end{array}\right),
$$

The characteristic equation of $L_{n}(\alpha)$ is

$$
\mu^{2}-\mu T_{n}(\alpha)+D_{n}(\alpha)=0, \quad n=0,1,2, \ldots,
$$

where

$$
\begin{aligned}
& T_{n}(\alpha)=-(D+1) \delta \frac{n^{2}}{l^{2}}+\alpha+\beta \\
& D_{n}(\alpha)=\frac{n^{4}}{l^{4}} D \delta^{2}-\frac{n^{2}}{l^{2}} \delta(\alpha+D \beta)+\alpha \beta+\alpha
\end{aligned}
$$


and the eigenvalues $\mu(\alpha)$ of $L_{n}(\alpha)$ are given by

$$
\begin{array}{r}
\mu(\alpha)=\frac{T_{n}(\alpha) \pm \sqrt{T_{n}^{2}(\alpha)-4 D_{n}(\alpha)}}{2}, \\
n=0,1,2, \ldots
\end{array}
$$

We identify the Hopf bifurcation value $\alpha$ satisfying the condition for Hopf bifurcation [Yi et al., 2009], which takes the following form:

$\left(\mathrm{H}_{1}\right)$ There exists $n \in \mathbb{N}_{0}$, such that

$$
\begin{aligned}
& T_{n}(\alpha)=0, \quad D_{n}(\alpha)>0, \\
& T_{j}(\alpha) \neq 0, \quad D_{j}(\alpha) \neq 0, \quad \text { for any } j \neq n .
\end{aligned}
$$

Let the unique pair of complex eigenvalues near the imaginary axis be $\gamma(\alpha) \pm i \omega(\alpha)$, then the following transversality condition holds:

$$
\gamma^{\prime}(\alpha) \neq 0
$$

We define a function

$$
\alpha^{H}(p)=(D+1) \delta p-\beta
$$

and for $j \in \mathbb{N}_{0}$, define

$$
\alpha_{j}^{H}=\alpha^{H}\left(\frac{j^{2}}{l^{2}}\right)=(D+1) \delta \frac{j^{2}}{l^{2}}-\beta,
$$

then $T_{j}\left(\alpha_{j}^{H}\right)=0$ and $T_{i}\left(\alpha_{j}^{H}\right) \neq 0$ for $i \neq j$. Since we require $\alpha<1$, then there is an $n_{0} \in \mathbb{N}$ such that $\alpha_{n_{0}}^{H}<1<\alpha_{n_{0}+1}^{H}$. Define

$$
l_{n}=n \sqrt{\frac{(D+1) \delta}{1+\beta}}, \quad n \in \mathbb{N}_{0} .
$$

Then for $l_{n}<l \leq l_{n+1}$, we have exactly $n+1$ potential Hopf bifurcation points $\alpha=\alpha_{j}^{H}(0 \leq j \leq n)$ defined by (11) and these points satisfy

$$
\alpha_{0}^{H}(=-\beta)<\alpha_{1}^{H}<\cdots<\alpha_{n}^{H}<1 .
$$

Next we only need to verify whether $D_{i}\left(\alpha_{j}^{H}\right) \neq 0$ for all $i \in \mathbb{N}_{0}$, and in particular, $D_{j}\left(\alpha_{j}^{H}\right)>0$. We claim that if $0>\beta>\frac{1}{D}-\frac{2}{\sqrt{D}}$, then $D_{i}\left(\alpha_{j}^{H}\right)>0$ for any $i \in \mathbb{N}_{0}$ and $\alpha_{j}^{H} \in(0,1)$. Indeed from $0<\alpha_{j}^{H}<1$, we have

$$
\begin{aligned}
D_{i}\left(\alpha_{j}^{H}\right) & =p_{i}^{2} D \delta^{2}-p_{i} \delta\left(\alpha_{j}^{H}+D \beta\right)+\alpha_{j}^{H} \beta+\alpha_{j}^{H} \\
& =p_{i}^{2} D \delta^{2}+\alpha_{j}^{H}\left(1+\beta-p_{i} \delta\right)-p_{i} \delta D \beta .
\end{aligned}
$$

If $0<p_{i} \leq \frac{1+\beta}{\delta}$, then $1+\beta-p_{i} \delta \geq 0$ and $D_{i}\left(\alpha_{j}^{H}\right)>0$. If $p_{i}>\frac{1+\beta}{\delta}$, and $0>\beta>\frac{1}{D}-\frac{2}{\sqrt{D}}$, then

$$
\begin{aligned}
D_{i}\left(\alpha_{j}^{H}\right) & >p_{i}^{2} D \delta^{2}+\left(1+\beta-p_{i} \delta\right)-p_{i} \delta D \beta \\
& =p_{i}^{2} D \delta^{2}-p_{i} \delta(1+D \beta)+1+\beta \\
& \geq-\frac{(1+D \beta)^{2}}{4 D}+1+\beta \\
& =\frac{1}{4 D}(2 \sqrt{D}-1+D \beta)(2 \sqrt{D}+1-D \beta) \\
& >0 .
\end{aligned}
$$

Finally let the eigenvalues close to the pure imaginary ones at $\alpha=\alpha_{j}^{H}$ be $\gamma(\alpha) \pm i \omega(\alpha)$. Then $\gamma^{\prime}\left(\alpha_{j}^{H}\right)=\frac{T_{j}^{\prime}\left(\alpha_{j}^{H}\right)}{2}=\frac{1}{2}>0$. Now by using the Hopf bifurcation theorem in [Yi et al., 2009], we can obtain the main result in this section.

Theorem 1. Let $l_{n}$ as defined in (12) and assume that $l_{n}<l \leq l_{n+1}$ for some $n \in \mathbb{N}_{0}$. Suppose that $D$ and $\beta$ satisfy

$$
D>\frac{1}{4}, \quad \text { and } \quad \frac{1}{D}-\frac{2}{\sqrt{D}}<\beta<0 .
$$

Then for (2), there exist $n+1$ Hopf bifurcation points $\alpha_{j}^{H}(0 \leq j \leq n)$ defined by (11), satisfying

$$
\alpha_{0}^{H}(=-\beta)<\alpha_{1}^{H}<\alpha_{2}^{H}<\cdots<\alpha_{n}^{H}<1 .
$$

At each $\alpha=\alpha_{j}^{H}$, the system (2) undergoes a Hopf bifurcation, and the bifurcating periodic orbits near $(\alpha, u, v)=\left(\alpha_{j}^{H}, 0,0\right)$ can be parameterized as a $C^{\infty}$ curve $\{(\alpha(s), u(s), v(s)): s \in(0, \delta)\}$ for some small $\delta>0$, so that

$$
\left\{\begin{aligned}
\alpha(s)=\alpha_{j}^{H}+ & o(s), \\
u(s)(x, t)= & s\left(a_{n} e^{2 \pi i t / T(s)}+\overline{a_{n}} e^{-2 \pi i t / T(s)}\right) \\
& \times \cos \frac{n}{l} x+o(s), \\
v(s)(x, t)= & s\left(b_{n} e^{2 \pi i t / T(s)}+\overline{b_{n}} e^{-2 \pi i t / T(s)}\right) \\
& \times \cos \frac{n}{l} x+o(s),
\end{aligned}\right.
$$

where $\left(a_{n}, b_{n}\right)$ is the corresponding eigenvector, and $T(s)=2 \pi / \sqrt{D_{j}\left(\alpha_{j}^{H}\right)}+o(s)\left(D_{j}\right.$ is defined in $\left.(8)\right)$. 


\section{Furthermore}

(1) The bifurcating periodic orbits from $\alpha=\alpha_{0}^{H}=$ $-\beta$ are spatially homogeneous, which coincide with the periodic orbits of the corresponding ODE system.

(2) The bifurcating periodic orbits from $\alpha=\alpha_{j}^{H}$ $(j \geq 1)$ are spatially nonhomogeneous.

Next we consider the bifurcation direction $\left(\alpha^{\prime}(0)>0(<0)\right)$ and stability of the bifurcating periodic orbits bifurcating from $\alpha=\alpha_{0}^{H}$ according to [Yi et al., 2009].

Theorem 2. For system (2), when $-1<\beta<0$, the Hopf bifurcation at $\alpha_{0}^{H}=-\beta$ is supercritical. That is, for a small $\varepsilon>0$ and $\alpha \in\left(\alpha_{0}^{H}, \alpha_{0}^{H}+\varepsilon\right)$, there is a small amplitude spatially homogenous periodic orbit, and this periodic orbit is locally asymptotically stable if (13) is satisfied.

Proof. Here we follow the notations and calculations in [Yi et al., 2009]. When $\alpha=\alpha_{0}^{H}=-\beta$, Eq. (7) has a pair of purely imaginary eigenvalues $\mu= \pm i \sqrt{-\beta-\beta^{2}}$ satisfying

$$
L_{0} q=i \sqrt{-\beta-\beta^{2}} q
$$

and we can choose $q=\left(a_{0}, b_{0}\right)^{T}=(-1,-\beta-$ $\left.i \sqrt{-\beta-\beta^{2}}\right)^{T}$. Define the inner product in $X_{\mathbb{C}}$ by

$$
\left\langle U_{1}, U_{2}\right\rangle=\int_{0}^{l \pi}\left(\overline{u_{1}} u_{2}+\overline{v_{1}} v_{2}\right) d x
$$

with $U_{i}=\left(u_{i}, v_{i}\right)^{T} \in X_{C}^{2}(i=1,2)$. We choose an associated eigenvector $q^{*}$ for the eigenvalue $\mu=-i \sqrt{-\beta-\beta^{2}}$ satisfying

$$
\begin{aligned}
& L_{0}^{*} q^{*}=-i \sqrt{-\beta-\beta^{2}} q^{*}, \\
& \left\langle q^{*}, q\right\rangle=1, \quad\left\langle q^{*}, \bar{q}\right\rangle=0,
\end{aligned}
$$

then

$$
\begin{aligned}
q^{*} & =\left(a_{0}^{*}, b_{0}^{*}\right)^{T} \\
& =\left(-\frac{1}{2 l \pi}+\frac{\beta i}{2 l \pi \sqrt{-\beta-\beta^{2}}}, \frac{-i}{2 l \pi \sqrt{-\beta-\beta^{2}}}\right)^{T} .
\end{aligned}
$$

Let $f(u, v)=\alpha u\left(1-r_{1} v^{2}\right)+v\left(1-r_{2} u\right), g(u$, $v)=v\left(\beta+\alpha r_{1} u v\right)+u\left(-\alpha+r_{2} v\right)$, then the partial derivatives for $f, g$ are evaluated as follows:

$$
\left\{\begin{aligned}
g_{u v} & =-f_{u v}, \quad g_{u v v}=-f_{u v v}, \\
f_{v v} & =f_{u u}=f_{v v v}=f_{u u v}=f_{u u u}=g_{u u u} \\
& =g_{v v}=g_{u u}=g_{u u v}=g_{v v v}=0 \\
f_{u v} & =-r_{2}, \quad f_{u v v}=-2 \alpha r_{1} .
\end{aligned}\right.
$$

By direct calculation, it follows that

$$
\left\{\begin{array}{l}
c_{0}=-2 r_{2}\left(\beta+i \sqrt{-\beta-\beta^{2}}\right), \\
d_{0}=-c_{0} \\
e_{0}=-2 \beta r_{2}, \\
f_{0}=-e_{0}, \\
g_{0}=2 \beta r_{1}\left(2 \beta^{2}+\beta-2 \beta i \sqrt{-\beta-\beta^{2}}\right), \\
h_{0}=-g_{0} .
\end{array}\right.
$$

Denote

$$
Q_{q, q}=\left(\begin{array}{c}
c_{n} \\
d_{n}
\end{array}\right), \quad Q_{q, \bar{q}}=\left(\begin{array}{c}
e_{0} \\
f_{0}
\end{array}\right), \quad C_{q, q, \bar{q}}=\left(\begin{array}{c}
g_{0} \\
h_{0}
\end{array}\right) .
$$

Then

$$
\begin{aligned}
& \left\langle q^{*}, Q_{q q}\right\rangle=c_{0}\left(-\frac{1}{2}-\frac{(\beta+1) i}{2 \sqrt{-\beta-\beta^{2}}}\right), \\
& \left\langle\bar{q}^{*}, Q_{q q}\right\rangle=c_{0}\left(-\frac{1}{2}+\frac{(\beta+1) i}{2 \sqrt{-\beta-\beta^{2}}}\right), \\
& \left\langle q^{*}, Q_{q \bar{q}}\right\rangle=e_{0}\left(-\frac{1}{2}-\frac{(\beta+1) i}{2 \sqrt{-\beta-\beta^{2}}}\right), \\
& \left\langle\bar{q}^{*}, Q_{q \bar{q}}\right\rangle=e_{0}\left(-\frac{1}{2}+\frac{(\beta+1) i}{2 \sqrt{-\beta-\beta^{2}}}\right), \\
& \left\langle q^{*}, C_{q q \bar{q}}\right\rangle=g_{0}\left(-\frac{1}{2}-\frac{(\beta+1) i}{2 \sqrt{-\beta-\beta^{2}}}\right) .
\end{aligned}
$$

Hence

$$
\begin{aligned}
H_{20}= & \left(c_{0}, d_{0}\right)^{T}-\left\langle q^{*}, Q_{q q}\right\rangle\left(a_{0}, b_{0}\right)^{T} \\
& -\left\langle\bar{q}^{*}, Q_{q q}\right\rangle\left(\overline{a_{0}}, \overline{b_{0}}\right)^{T}, \\
= & c_{0}(0,-1-\beta)^{T}+c_{0}(0,1+\beta)^{T}=0, \\
H_{11}= & \left(e_{0}, f_{0}\right)^{T}-\left\langle q^{*}, Q_{q \bar{q}}\right\rangle\left(a_{0}, b_{0}\right)^{T} \\
& -\left\langle\bar{q}^{*}, Q_{q \bar{q}}\right\rangle\left(\overline{a_{0}}, \overline{b_{0}}\right)^{T}, \\
= & e_{0}(0,-1-\beta)^{T}+e_{0}(0,1+\beta)^{T}=0,
\end{aligned}
$$




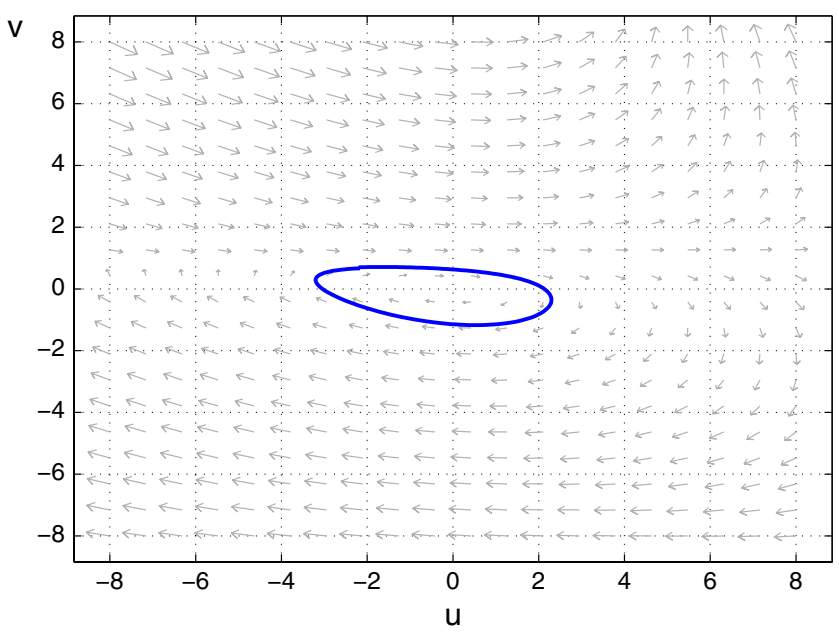

(a)

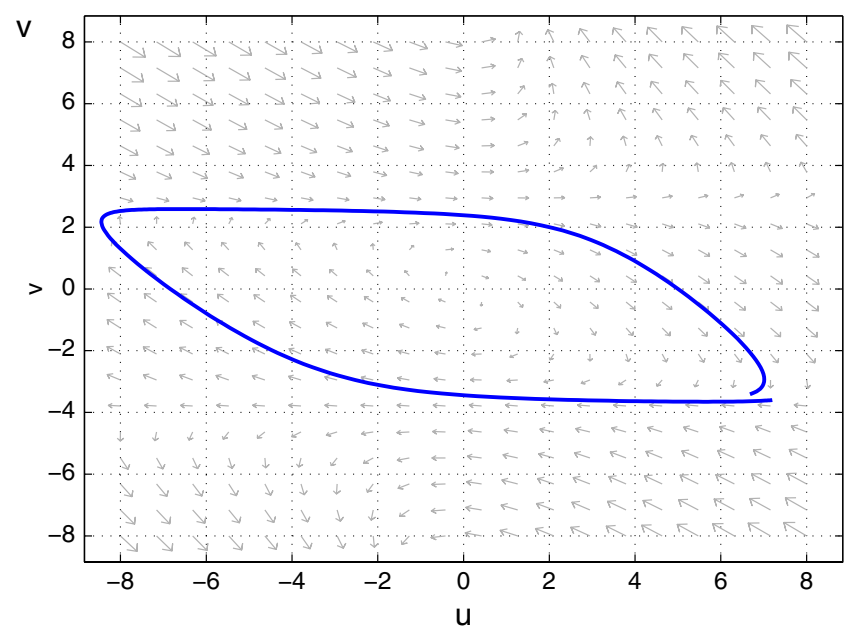

(b)

Fig. 1. Phase portraits for the ODE system corresponding to (2) when $\beta=-0.1, r_{1}=0.1, r_{2}=0.1$, The horizontal axis is $u$, and the vertical axis is $v$. (a) $\alpha=0.12$, one small amplitude limit cycle and (b) $\alpha=0.9$, a large amplitude limit cycle. Here the Hopf bifurcation point $\alpha_{0}^{H}=0.1$.

which implies that $\omega_{20}=\omega_{11}=0$, then

$$
\left\langle q^{*}, Q_{\omega_{11} q}\right\rangle=\left\langle q^{*}, Q_{\omega_{20} \bar{q}}\right\rangle=0 .
$$

Therefore

$$
\begin{aligned}
\operatorname{Re} & \left(c_{1}\left(\alpha_{0}^{H}\right)\right) \\
& =\operatorname{Re}\left\{\frac{i}{2 \omega_{0}}\left\langle q^{*}, Q_{q q}\right\rangle \cdot\left\langle q^{*}, Q_{q \bar{q}}\right\rangle+\frac{1}{2}\left\langle q^{*}, C_{q q \bar{q}}\right\rangle\right\} \\
& =\frac{\beta^{2} r_{2}^{2}+\beta r_{2}^{2}}{2\left(-\beta-\beta^{2}\right)}-\frac{6 \beta^{2} r_{1} \sqrt{-\beta-\beta^{2}}}{4 \sqrt{-\beta-\beta^{2}}} \\
& =-\frac{r_{2}^{2}+3 \beta^{2} r_{1}}{2}<0 .
\end{aligned}
$$

Moreover $T^{\prime}\left(\alpha_{j}^{H}\right)=1>0$, therefore when $\alpha>$ $\alpha_{0}^{H}=-\beta$, the equilibrium point of (2) is unstable, and the system must have a periodic orbit by the Poincaré-Bendixson theorem. From the calculation above, the Hopf bifurcation at $\alpha=\alpha_{0}^{H}$ is supercritical; and when $\alpha \in\left(\alpha_{0}^{H}, \alpha_{0}^{H}+\varepsilon\right)$, the bifurcating periodic orbit is locally asymptotically stable if (13) is satisfied.

Note that the bifurcating periodic orbit may not be stable if a Turing bifurcation occurs at some $\alpha<-\beta$ (see Sec. 3). The periodic orbits of system (2) for some parameters are shown in Fig. 1, and a bifurcation diagram of the periodic orbits is shown in Fig. 2.

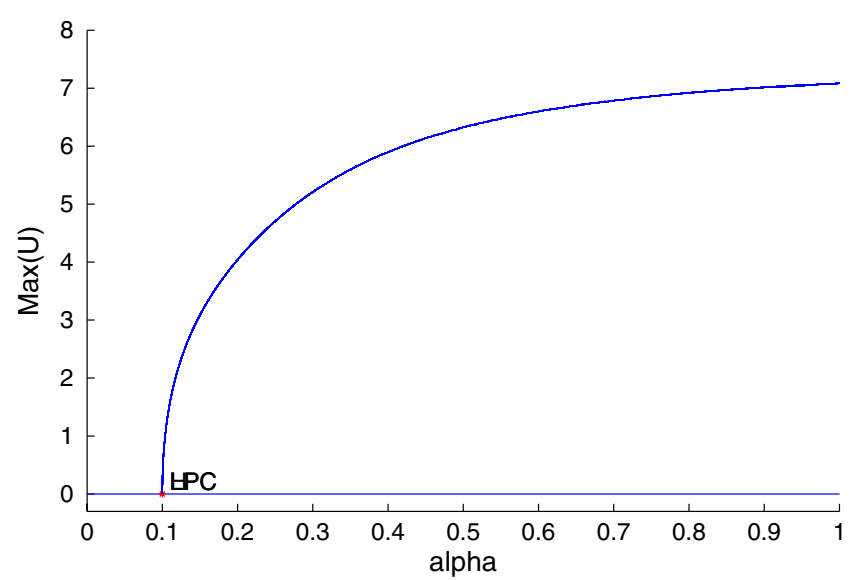

Fig. 2. Bifurcation of periodic orbits for the ODE system corresponding to $(2) \beta=-0.1, r_{1}=0.1, r_{2}=0.1$. The horizontal axis is $\alpha$, and the vertical axis is $\max u(t)$ for the limit cycle $(u(t), v(t))$. Here the Hopf bifurcation point $\alpha_{0}^{H}=0.1$.

\section{Steady State Bifurcation Analysis}

In this section, we consider the steady state solutions of system (2). We consider the equations:

$$
\left\{\begin{array}{r}
D \delta u^{\prime \prime}+\alpha u\left(1-r_{1} v^{2}\right)+v\left(1-r_{2} u\right)=0, \\
x \in(0, l \pi), \\
\delta v^{\prime \prime}+v\left(\beta+\alpha r_{1} u v\right)+u\left(-\alpha+r_{2} v\right)=0, \\
x \in(0, l \pi), \\
u^{\prime}(0)=v^{\prime}(0)=u^{\prime}(l \pi)=v^{\prime}(l \pi)=0 .
\end{array}\right.
$$

Recall $D_{n}(\alpha)$ and $T_{n}(\alpha)$ defined in (8). Now we identify steady state bifurcation value $\alpha$ of 
steady state system (20), which satisfies the following steady state bifurcation condition:

$\left(\mathrm{H}_{2}\right)$ There exists $n \in \mathbb{N}_{0}$ such that

$$
\begin{aligned}
& D_{n}(\alpha)=0, \quad T_{n}(\alpha) \neq 0, \\
& D_{j}(\alpha) \neq 0, \quad T_{j}(\alpha) \neq 0, \quad \text { for any } j \neq n ;
\end{aligned}
$$

and

$$
\frac{d}{d \alpha} D_{n}(\alpha) \neq 0
$$

Clearly $D_{0}(\alpha)=\alpha \beta+\alpha>0$ for $-1<\beta<0$ and $\alpha>0$, hence we only consider the bifurcation mode $n \in \mathbb{N}$. We fix $-1<\beta<0$ to determine a bifurcation value $\alpha$ satisfying condition $\left(\mathrm{H}_{2}\right)$. We notice that $D_{n}(\alpha)=0$ is equivalent to

$$
D(\alpha, p):=(\alpha-D \delta p)(\beta-\delta p)+\alpha=0,
$$

where $p=\frac{n^{2}}{l^{2}}$. Solving $\alpha$ from (23), we have

$$
\begin{aligned}
\alpha^{S}(p) & =\frac{D \delta p(\beta-\delta p)}{\beta+1-\delta p} \\
& =p D \delta\left(1+\frac{1}{\delta p-(\beta+1)}\right) .
\end{aligned}
$$

We also solve $p$ from (23), and we obtain

$$
\begin{aligned}
p & =p_{ \pm}(\alpha) \\
& :=\frac{(\alpha+D \beta) \pm \sqrt{(\alpha+D \beta)^{2}-4 D \alpha(1+\beta)}}{2 D \delta} .
\end{aligned}
$$

Define

$$
\tilde{l}_{n}=n \sqrt{\frac{\delta}{\beta+1}}, \quad n=1,2, \ldots,
$$

then for any $0<l<\tilde{l}_{n}$, there exist a unique $\alpha_{n}^{S}:=\alpha^{S}\left(\frac{n^{2}}{l^{2}}\right)$ such that $D_{n}\left(\alpha_{n}^{S}\right)=0$, where $\alpha^{S}(\cdot)$ is defined in (24). These points $\alpha_{n}^{S}$ are potential steady state bifurcation points.

The function $\alpha^{S}(p)$ and the functions $p_{ \pm}(\alpha)$ satisfy the following properties.

Lemma 1. Define

$$
\begin{aligned}
p_{*} & =\frac{1}{\delta}(\sqrt{\beta+1}+\beta+1), \\
\alpha_{*} & =\alpha\left(p_{*}\right)=D(\sqrt{\beta+1}+1)^{2} .
\end{aligned}
$$

Then the function $\alpha^{S}:\left(\frac{\beta+1}{\delta}, \infty\right) \rightarrow \mathbb{R}^{+}$defined in (24) has a unique critical point $p_{*} \in\left(\frac{\beta+1}{\delta}, \infty\right)$, which is the global minimum of $\alpha^{S}(p)$ on $\left(\frac{\beta+1}{\delta}, \infty\right)$, and $\lim _{p \rightarrow\left(\frac{\beta+1}{\delta}\right)^{+}} \alpha^{S}(p)=\lim _{p \rightarrow \infty} \alpha^{S}(p)=\infty$. Consequently for $\alpha \geq \alpha_{*}:=\alpha^{S}\left(p_{*}\right), p_{ \pm}(\alpha)$ are well defined as in (25); $p_{+}(\alpha)$ is monotone increasing and $p_{-}(\alpha)$ is monotone decreasing; $\sup _{\alpha>\alpha_{*}} \times$ $p_{+}(\alpha)=\lim _{\alpha \rightarrow \infty} p_{+}(\alpha)=\infty, \inf _{\alpha>\alpha_{*}} p_{-}(\alpha)=$ $\lim _{\alpha \rightarrow \infty} p_{-}(\alpha)=\frac{\beta+1}{\delta}$, and $p_{+}\left(\alpha_{*}\right)=p_{-}\left(\alpha_{*}\right)=p_{*}$.

Proof. Let $D(\alpha, p)$ be defined as in (23). Then the set $\Lambda:=\{(\alpha, p): \alpha>0, p>0\}$ is given by the curve $\left\{\left(\alpha^{S}(p), p\right): \frac{\beta+1}{\delta}<p<\infty\right\}$. Next we prove that $\alpha^{S}(p)$ has a unique critical point. Differentiating $D\left(\alpha^{S}(p), p\right)=0$ twice and letting $\left(\alpha^{S}\right)^{\prime}(p)=0$, we have

$$
\begin{aligned}
\frac{d^{2}}{d p^{2}} D\left(\alpha^{S}(p), p\right) & =(\beta+1-\delta p)\left(\alpha^{S}\right)^{\prime \prime}(p)+2 D \delta^{2} \\
& =0 .
\end{aligned}
$$

Thus

$$
\left(\alpha^{S}\right)^{\prime \prime}(p)=\frac{2 D \delta^{2}}{\delta p-(\beta+1)}>0 .
$$

Therefore for any critical point $p$ of $\alpha^{S}(p)$, we must have $\left(\alpha^{S}\right)^{\prime \prime}(p)>0$, and thus the critical point of $\alpha^{S}(p)$ must be unique and be a local minimum point.

Since we have $\lim _{p \rightarrow\left(\frac{\beta+1}{\delta}\right)^{+}} \alpha^{S}(p)=\lim _{p \rightarrow \infty} \times$ $\alpha^{S}(p)=\infty$, then the unique critical point $p_{*}$ of $\alpha^{S}(p)$ is the global minimum point. Since $(25)$ is also obtained by solving (23), then $\Lambda=\left\{\left(\alpha^{S}(p), p\right)\right.$ : $\left.\frac{\beta+1}{\delta}<p<\infty\right\}$ and the curves $\left(\alpha, p_{ \pm}(\alpha)\right)$ are identical. Then the properties of $\alpha^{S}(p)$ determine the monotonicity and limiting behavior of $p_{ \pm}(\alpha)$.

From Lemma 1, it is possible that $\alpha\left(p_{i}\right)=\alpha\left(p_{j}\right)$ and $p_{-}\left(\alpha_{i}^{S}\right)=p_{+}\left(\alpha_{j}^{S}\right)$, for some $i, j(i<j)$. In this case, for $\alpha=\alpha_{i}^{S}=\alpha_{j}^{S}, 0$ is not a simple eigenvalue of $L(\alpha)$, so we shall not consider bifurcations at such points. From the properties of $p_{ \pm}(\alpha)$ in Lemma 1, we know the multiplicity of 0 as eigenvalue of $L(\alpha)$ is at most 2. On the other hand, it is also possible that some $\alpha_{i}^{S}=\alpha_{j}^{H}$, so the dimension of center manifold of the equilibrium $\left(u_{\alpha}, v_{\alpha}\right)$ can be between 1 to 4 .

We claim that there are only countably many $l>0$, in fact only finitely many $l \in(0, M)$ for any given $M>0$, such that $\alpha=\alpha_{i}^{S}=\alpha_{j}^{S}$ or $\alpha_{i}^{S}=\alpha_{j}^{H}$, for $i, j \in \mathbb{N}$. Let $E_{n}(\alpha, l)=l^{4} D_{n}(\alpha), F_{n}(\alpha, l)=$ $l^{2} T_{n}(\alpha)$. Then for any $n \in \mathbb{N}, E_{n}(\alpha, l)$ and $F_{n}(\alpha, l)$ 
are polynomials of $\alpha, l$ with real coefficients. Hence on $(\alpha, l)$-plane, the set $q_{n}=\left\{(\alpha, l): E_{n}(\alpha, l)=0\right\}$, or $p_{n}=\left\{(\alpha, l): F_{n}(\alpha, l)=0\right\}$ is the union of countable analytic curves. Moreover, we require $\alpha \in$ $\left[\alpha_{*}, \infty\right)$, then for any $M>0$, there are only finitely many $i, j \in \mathbb{N}$, such that $q_{i} \cap\left(\left[\alpha_{*}, \infty\right) \times[0, M]\right) \neq \emptyset$ and $p_{j} \cap\left(\left[\alpha_{*}, \infty\right) \times[0, M]\right) \neq \emptyset$. These finitely many $q_{i}, p_{j}$ only have finitely many intersection points in $\left[\alpha_{*}, \infty\right) \times[0, M]$ due to the analyticity, and thus the intersection points of different $q_{i}, p_{j}$ in $\left[\alpha_{*}, \infty\right) \times[0, \infty]$ are countable. Define

$$
\begin{aligned}
L^{E}= & \left\{l>0: E_{i}(\alpha, l)=E_{j}(\alpha, l)\right. \text { or } \\
& \left.E_{i}(\alpha, l)=F_{j}(\alpha, l), \alpha \in\left[\alpha_{*}, \infty\right), i, j \in \mathbb{N}\right\} .
\end{aligned}
$$

Then the points $L^{E}$ can be arranged as a sequence whose only limit point is $\infty$.

Hence if $l \in \mathbb{R}^{+} \backslash L^{E}$, and $\alpha_{j}^{S}$ is well defined, then $\left(\mathrm{H}_{2}\right)$ is satisfied at $\alpha=\alpha_{j}^{S}$. Now we show that $\frac{d}{d \alpha} D_{j}\left(\alpha_{j}^{S}\right) \neq 0$. By direct calculation, we have

$$
\frac{d}{d \alpha} D_{j}\left(\alpha_{j}^{S}\right)=\beta+1-\delta p_{j}<0, \quad \text { where } p_{j}=\frac{j^{2}}{l^{2}} .
$$

Summarizing the above discussions, and using a general bifurcation theorem [Shi \& Wang, 2009; Yi et al., 2009], we obtain the main result of this section on the global bifurcations of steady state solutions:

Theorem 3. Assume that

$$
-1<\beta<\frac{1}{D}-\frac{2}{\sqrt{D}},
$$

and let $n \in \mathbb{N}$. Suppose that $l \in(0, \infty) \backslash L^{E}$, and $\tilde{l}_{n-1}<l<\tilde{l}_{n}$ for some $n \in \mathbb{N}$, where $\tilde{l}_{n}$ is defined in (26) and $L^{E}$ is a countable subset of $\mathbb{R}^{+}$defined in (28). Then $\alpha_{n}^{S}=\alpha^{S}\left(\frac{n^{2}}{l^{2}}\right)$ satisfies $\alpha_{*}<\alpha_{n}^{S}<1$, and $\alpha=\alpha_{n}^{S}$ is a bifurcation point for (20). Moreover,

(1) There exists a $C^{\infty}$ smooth curve $\Gamma_{j}$ of solutions of (20) bifurcating from $(\alpha, u, v)=\left(\alpha_{j}^{S}, 0,0\right)$, with $\Gamma_{j}$ contained in a global branch $\mathcal{C}_{j}$ of solutions of (20).

(2) Near $(\alpha, u, v)=\left(\alpha_{j}^{S}, 0,0\right), \Gamma_{j}=\left\{\left(\alpha_{j}(s), u_{j}(s)\right.\right.$, $\left.\left.v_{j}(s)\right): s \in(-\epsilon, \epsilon)\right\}$, where $u_{j}(s)=s a_{j} \cos (j x /$ $l)+s \psi_{1, j}(s), v_{j}(s)=s b_{j} \cos (j x / l)+s \psi_{2, j}(s)$, $s \in(-\epsilon, \epsilon)$, for some $C^{\infty}$ smooth functions $\alpha_{j}$, $\psi_{1, j}, \psi_{2, j}$ such that $\alpha_{j}(0)=\alpha_{j}^{S}$ and $\psi_{1, j}(0)=$ $\psi_{2, j}(0)=0$; Here $a_{j}$ and $b_{j}$ satisfy

$$
L\left(\alpha_{j}^{S}\right)\left[\left(a_{j}, b_{j}\right)^{T} \cos \left(\frac{n x}{l}\right)\right]=(0,0)^{T} .
$$

(3) Either $C_{j}$ contains another $\left(\alpha_{m}^{S}, 0,0\right)$ for $m \neq j$, or $C_{j}$ is unbounded.

Proof. To apply Theorem 3.2 in [Yi et al., 2009], we only need to show the local conditions $\left(\mathrm{H}_{2}\right)$ and $\frac{d}{d \alpha} D_{j}\left(\alpha_{j}^{S}\right) \neq 0$, which have been proved in the previous paragraphs. Note that we exclude $L^{E}$, so $\alpha=\alpha_{j}^{S}$ is always a bifurcation from a simple eigenvalue point. Thus the results follow from Theorem 3.2 in [Yi et al., 2009].

Note that $l \notin L^{E}$ is only technical, and for $l \in L^{E}$, as long as the bifurcation value is simple, then the bifurcation result still holds. We also remark that at each bifurcation point $\alpha=\alpha_{j}^{S}$, the steady state bifurcation is a pitchfork one so that $\alpha_{j}^{\prime}(0)=0$. This is natural since $(u(l \pi-x), v(l \pi-x))$ is also a solution if $(u(x), v(x))$ is one. Thus $\alpha_{j}^{\prime \prime}(0)$ determines the direction of the bifurcation. The value $\alpha_{j}^{\prime \prime}(0)$ can be calculated as in [Jin et al., 2013], so one can determine whether it is a supercritical or subcritical pitchfork bifurcation.

\section{Numerical Simulations and Discussion}

In Secs. 2 and 3, we consider the instability of the unique positive constant steady state $(0,0)$ of $(2)$ and related bifurcation phenomena. In the analysis, we fix parameters $D, \delta, \beta, r_{1}, r_{2}$, and the length parameter $l$, and use $\alpha$ as the bifurcation parameter.

For (2) we have identified two critical parameter values for the system (2): $\alpha=-\beta$, which is the smallest Hopf bifurcation point, and $\alpha=\alpha_{*}$ (defined as in (27)). The constant equilibrium $(0,0)$ is locally asymptotically stable when $\alpha<$ $\min \left\{-\beta, \alpha_{*}\right\}$. When $-\beta<\alpha_{*}$, then $(0,0)$ loses the stability at $\alpha=-\beta$ through a Hopf bifurcation; when $\alpha_{*}<-\beta$, a steady state bifurcation is likely to happen for some $\alpha \in\left(\alpha_{*}, 1\right)$. Figure 3(a) shows the graph of $T(\alpha, p)=0$ and $D(\alpha, p)=0$ with a set of parameters so that $-\beta<\alpha_{*}$, while Fig. 3(b) shows the one for the case $\alpha_{*}<-\beta$.

In the second case, one can choose $l$ so that there are steady state bifurcation points in the interval $\left(\alpha_{*}, 1\right)$. For example, for the parameters 


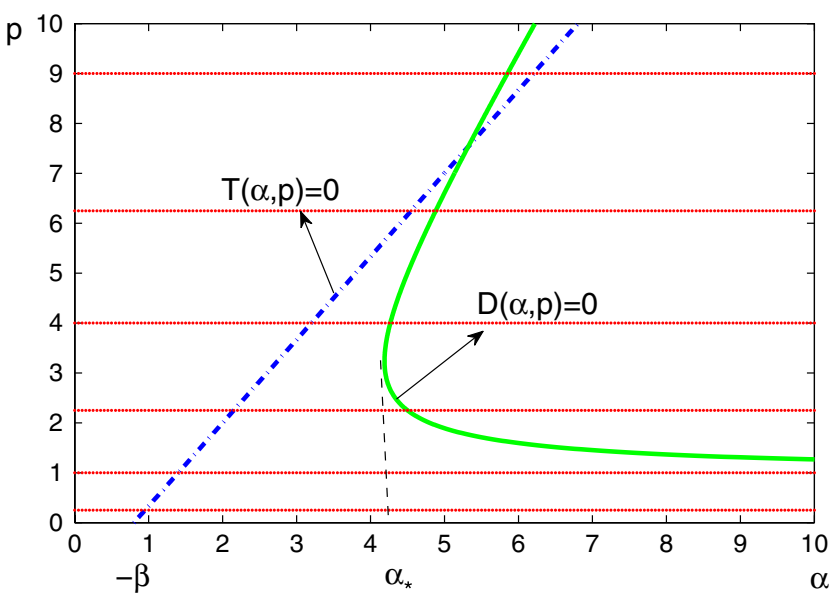

(a)

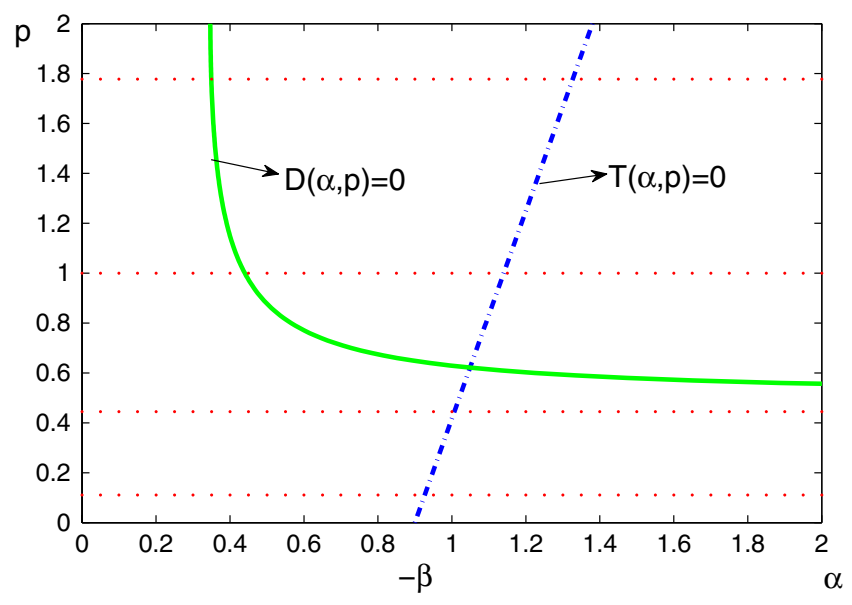

(b)

Fig. 3. Graph of $T(\alpha, p)=0$ and $D(\alpha, p)=0$. (a) $\beta=-0.8, D=2, \delta=0.2$ and $l=2$; (b) $\beta=-0.9, D=0.2, \delta=0.2$ and $l=3$. The horizontal lines are $p=n^{2} / l^{2}$.

given in Fig. 3(b), if we choose $l=3$, then we have

$$
\begin{aligned}
\alpha_{4}^{S} & =0.349<\alpha_{5}^{S}=0.355<\alpha_{6}^{S}=0.389<\alpha_{7}^{S} \\
& =0.438<\alpha_{3}^{S}=0.44<\alpha_{8}^{S}=0.5<\alpha_{0}^{H}=0.9
\end{aligned}
$$

We use several numerical simulations to illustrate and complement our analytical results. For the parameters given in Fig. 3(a) with $l=2$, a simulation with $\alpha=0.9>\alpha_{0}^{H}=0.8$ is shown in Fig. 4, and a spatially homogeneous limit cycle is the asymptotical limit here.

On the other hand, for the parameters given in Fig. 3(b) with $l=3$, several steady state bifurcations occur at $\alpha$-values smaller than $\alpha_{0}^{H}$ [see (31)]. Figures 5 and 6 show two solutions with different initial conditions. While each shows a stationary spatial pattern, the one in Fig. 5 corresponds to constant steady state; while the one in Fig. 6 appears to have spatial period $3 \pi / 2$, which corresponds to $n=4(\operatorname{mode} \cos (4 x / 3))$. From $(31)$, the parameter $\alpha=0.35$ in Figs. 5 and 6 is between $\alpha_{5}^{S}=0.355$ and $\alpha_{4}^{S}=0.349$. Figures 5 and 6 show that there is a bistability between the constant steady state solution and a nonconstant one with mode $n=4$.

Our analytical results in earlier sections and the numerical simulations guided by the analytical

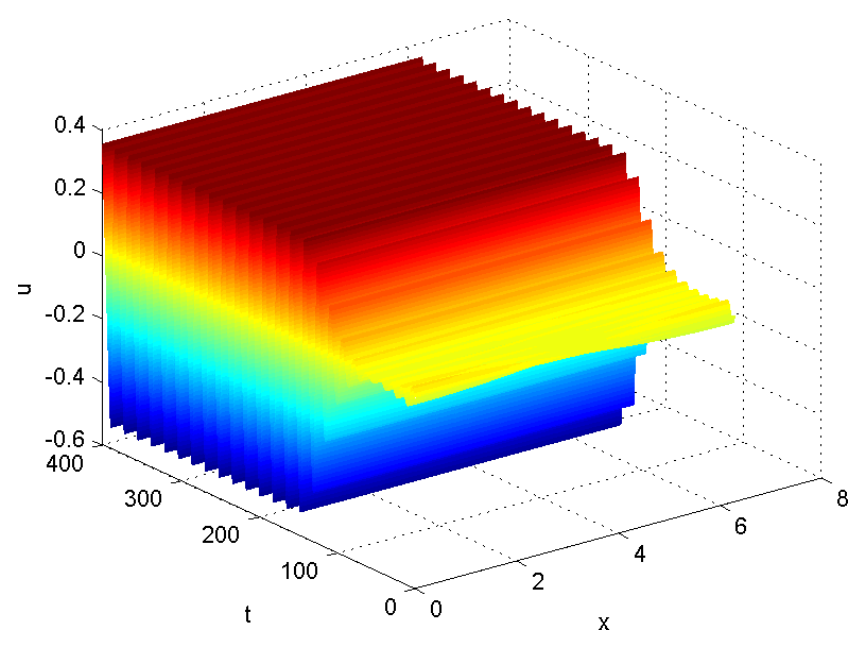

(a)

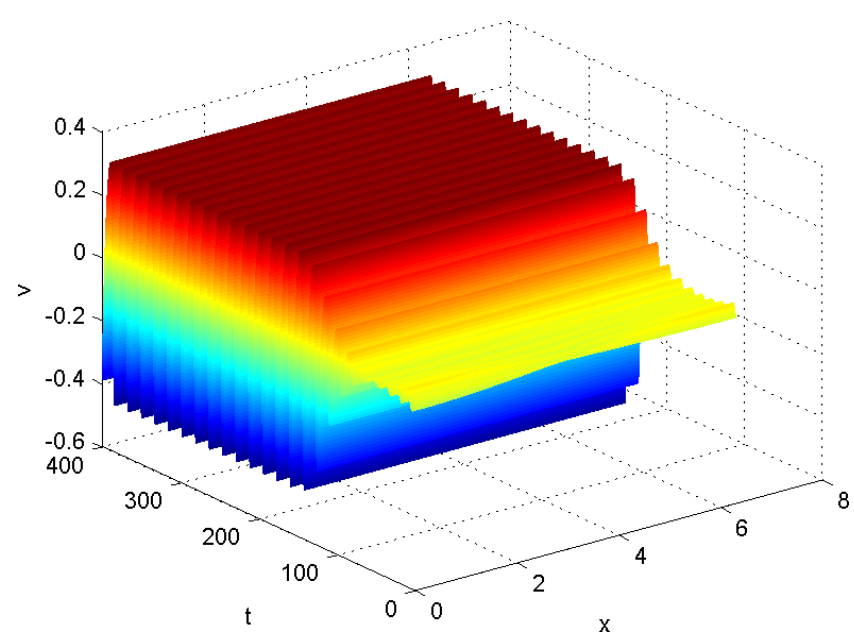

(b)

Fig. 4. Numerical simulation of the system (2). (a) $u(x, t)$ and (b) $v(x, t)$. Here $D=2, \alpha=0.9, \beta=-0.8, \delta=0.2, r_{1}=1$, $r_{2}=1, l=2,0 \leq t \leq 400$, and the initial values $u_{0}(x)=0.01 \cos (x / 2) ; v_{0}(x)=0.01 \cos (x / 2)$. The solution converges to a spatially homogenous periodic orbit. 


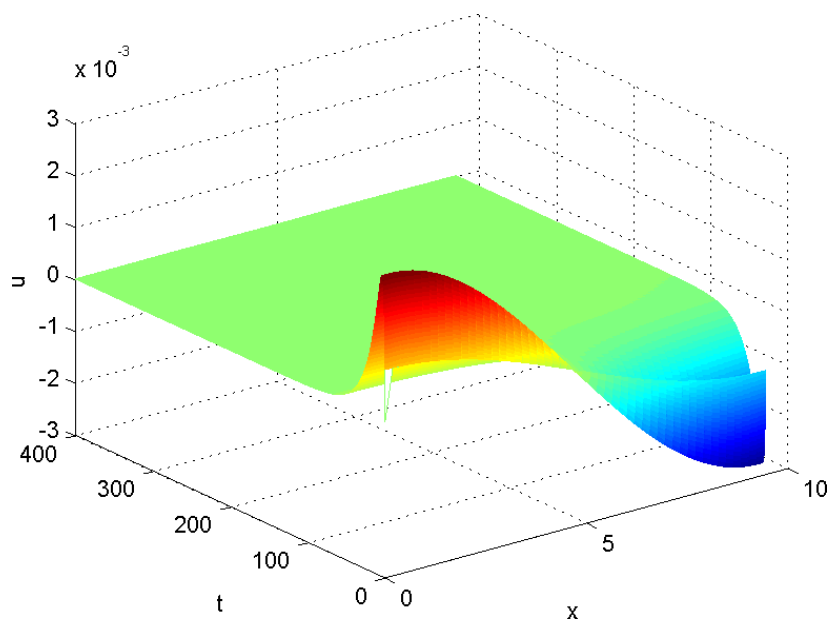

(a)

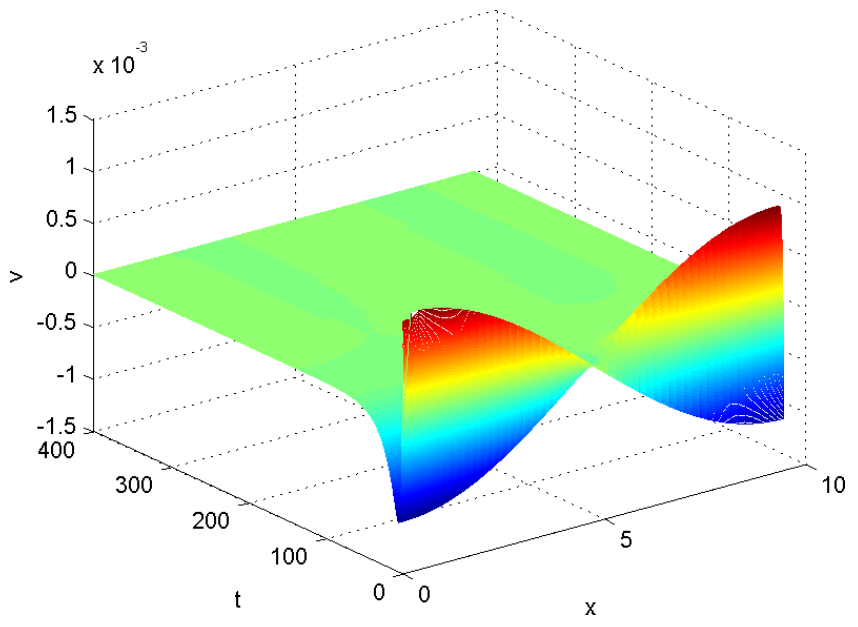

(b)

Fig. 5. Numerical simulation of system (2). (a) $u(x, t)$ and (b) $v(x, t)$. Here $D=0.2, \alpha=0.35, \beta=-0.8, \delta=0.2, r_{1}=1$, $r_{2}=1, l=2,0 \leq t \leq 400$, and the initial values $u_{0}(x)=0.001 \cos (x / 3) ; v_{0}(x)=0.001 \cos (x / 3)$. The solution converges to the constant steady state.

results show a rough picture of the dynamics in terms of system parameters $\alpha, \beta$ and $D$. There are several parameter regimes where the dynamical behavior of (2) are drastically different.

(1) When $0<-\beta<1<\alpha_{*}$ is satisfied, that is

$$
D>\frac{1}{4} \text { and } \frac{1}{D}-\frac{2}{\sqrt{D}}<\beta<0
$$

then there are a sequence of Hopf bifurcation points $-\beta=\alpha_{0}^{H}<\alpha_{1}^{H}<\cdots<\alpha_{n}^{H}<1$ where periodic orbits of (2) bifurcate out from the constant steady state $(0,0)$ (see Theorem 1 ).

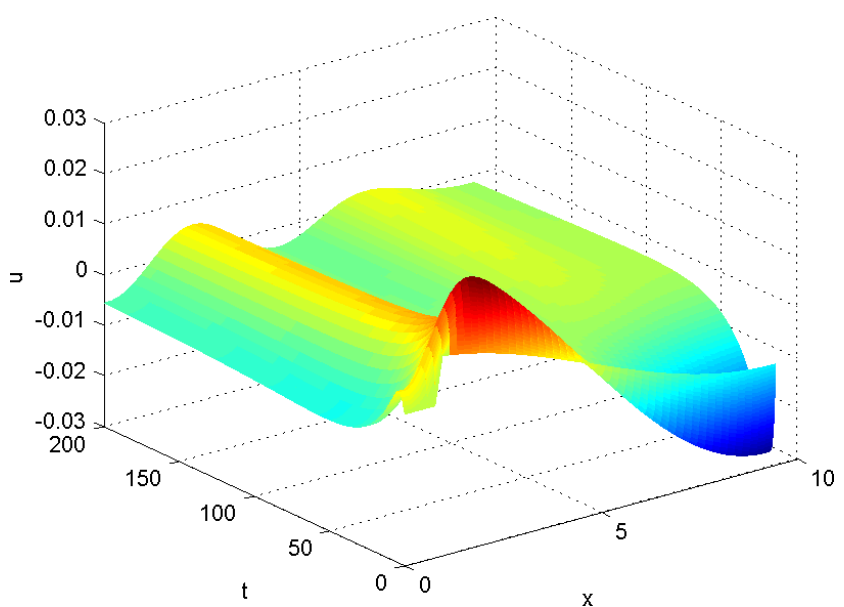

(a)
In particular, $(0,0)$ loses the local stability to a spatially homogenous periodic orbit at $\alpha=-\beta$. On the other hand, since $1<\alpha_{*}$, there is no steady state bifurcation for any $\alpha \in(0,1)$. Hence the parameter regime given by (32) is dominated by time-periodic patterns but probably not stationary spatially nonhomogeneous patterns. The number $n$ of spatially nonhomogeneous Hopf bifurcation points depends on $l$.

(2) If

$$
-1<\beta<\min \left\{\frac{1}{D}-\frac{2}{\sqrt{D}}, 0\right\}
$$

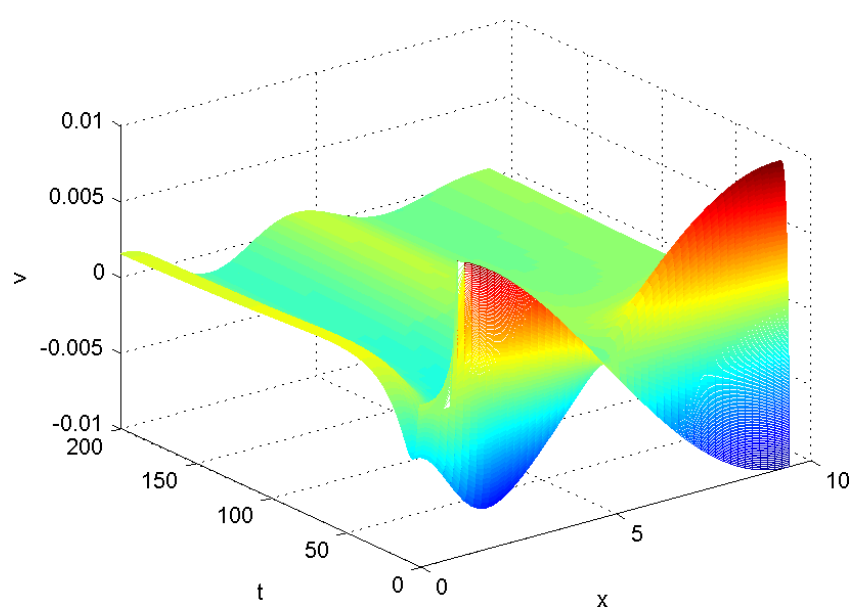

(b)

Fig. 6. Numerical simulation of system (2). (a) $u(x, t)$ and (b) $v(x, t)$. Here $D=0.2, \alpha=0.35, \beta=-0.8, \delta=0.2, r_{1}=1$, $r_{2}=1, l=2,0 \leq t \leq 200$, and the initial values $u_{0}(x)=0.01 \cos (5 x / 3) ; v_{0}(x)=0.01 \cos (5 x / 3)$. The solution converges to the constant steady state. 
then $0<\max \left\{\alpha_{*},-\beta\right\}<1$. In this case both the results in Theorem 1 and the ones in Theorem 3 are applicable. Hence possibly both Hopf bifurcations and steady state bifurcations occur for $\alpha \in\left(\min \left\{\alpha_{*},-\beta\right\}, 1\right)$, and these bifurcation points form an intertwining sequence of bifurcation points.

\section{Acknowledgment}

We thank two anonymous referees for helpful suggestions which improve the original version of the manuscript.

\section{References}

Aragón, J. L., Torres, M., Gil, D., Barrio, R. A. \& Maini, P. K. [2002] "Turing patterns with pentagonal symmetry," Phys. Rev. E 65, 051913.

Asai, R., Taguchi, E., Kume, Y., Saito, M. \& Kondo, S. [1999] "Zebrafish leopard gene as a component of the putative reaction-diffusion system," Mech. Dev. 89, 87-92.

Barrio, R. A., Varea, C., Aragón, J. L. \& Maini, P. K. [1999] "A two-dimensional numerical study of spatial pattern formation in interacting Turing systems," Bull. Math. Biol. 61, 483-505.

Barrio, R. A., Maini, P. K., Aragón, J. L. \& Torres, M. [2002] "Size-dependent symmetry breaking in models for morphogenesis," Physica D 168-169, 61-72.

Chen, S.-S., Shi, J.-P. \& Wei, J.-J. [2012] "Global stability and Hopf bifurcation in a delayed diffusive LeslieGower predator-prey system," Int. J. Bifurcation and Chaos 22, 1250061.

Chen, S.-S., Shi, J.-P. \& Wei, J.-J. [2013] "Time delay induced instabilities and Hopf bifurcations in general reaction-diffusion systems," J. Nonlin. Sci. 23, 1-38.

Crandall, M. G. \& Rabinowitz, P. H. [1971] "Bifurcation from simple eigenvalues," J. Funct. Anal. 8, 321-340.

Crandall, M. G. \& Rabinowitz, P. H. [1973] "Bifurcation perturbation of simple eigenvalues and linearized stability," Arch. Rat. Meth. Anal. 52, 161-180.

De Kepper, P., Castets, V. \& Dulos, E. [1991] "Turingtype chemical patterns in the chlorite-iodide-malonic acid reaction," Physica D 49, 161-169.

Gierer, A. \& Meinhardt, H. [1972] "A theory of biological pattern formation," Kybernetik 12, 30-39.

Gray, P. \& Scott, S. [1990] Chemical Oscillations and Instabilities: Nonlinear Chemical Kinectics (Clarendon Press, Oxford).

Han, W. \& Bao, Z. [2009] "Hopf bifurcation analysis of a reaction-diffusion Sel'kov system," J. Math. Anal. Appl. 356, 633-641.
Jin, J., Shi, J.-P., Wei, J.-J. \& Yi, F.-Q. [2013] "Bifurcations of patterned solutions in diffusive LengyelEpstein system of CIMA chemical reaction," Rocky Mount. J. Math. 43, 1637-1674.

Jung, H. S., Francis-West, P. H., Widelitz, R. B., Jiang, T. X., Ting-Berreth, S., Tickle, C., Wolpert, L. \& Chuong, C. M. [1998] "Local inhibitory action of BMPs and their relationships with activators in feather formation: Implications for periodic patterning," Dev. Biol. 196, 11-23.

Kondo, S. \& Asai, R. [1995] "A reaction-diffusion wave on the marine angelfish Pomacanthus," Nature 376, $765-768$.

Leppänen, T., Karttunen, M., Kaski, K., Barrio, R. A. \& Zhang, L. [2002] "A new dimension to Turing patterns," Physica D 168-169, 35-44.

Leppänen, T., Karttunen, M., Barrio, R. A. \& Kaski, K. [2004] "Morphological transitions and bistability in Turing systems," Phys. Rev. E 70, 066202.

Liu, J.-X., Yi, F.-Q. \& Wei, J.-J. [2010] "Multiple bifurcation analysis and spatiotemporal patterns in a 1-D Gierer-Meinhardt model of morphogenesis," Int. J. Bifurcation and Chaos 20, 1007-1025.

Liu, P., Shi, J.-P., Wang, Y.-W. \& Feng, X.-H. [2013] "Bifurcation analysis of reaction-diffusion Schnakenberg model," J. Math. Chem. 51, 2001-2019.

Meinhardt, H. [1982] Models of Biological Pattern Formation (Academic Press, London).

Meinhardt, H. [1995] The Algorithmic Beauty of Sea Shells (Springer, Berlin).

Murray, J. D. [1981] "A pre-pattern formation mechanism for animal coat markings," J. Theor. Biol. 88, 161-199.

Murray, J. D. [1982] "Parameter space for Turing instability in reaction-diffusion mechanisims: A comparison of models," J. Theoret. Biol. 98, 143-163.

Murray, J. D. [2002/03] Mathematical Biology, 3rd edition (Springer-Verlag, NY).

Ni, W.-M. \& Tang, M. [2005] "Turing patterns in the Lengyel-Epstein system for the CIMA reaction," Trans. Amer. Math. Soc. 357, 3953-3969.

Nicolis, G. \& Prigogine, I. [1977] Self-Organization in Nonequilibrium Systems (John Wiley and Sons, NY).

Ouyang, Q. \& Swinney, H. L. [1991] "Transition from a uniform state to hexagonal and striped Turing patterns," Nature 352, 610-612.

Painter, K. J., Maini, P. K. \& Othmer, H. G. [1999] "Stripe formation in juvenile Pomacanthus explained by a generalized Turing mechanism with chemotaxis," Proc. Natl. Acad. Sci. 96, 5549-5554.

Rudovics, B., Barillot, E. \& Davies, P. W. [1999] "Experimental studies and quantitative modeling of Turing patterns in the (chlorine dioxide, iodine, malonic acid) reaction," J. Chem. Phys. 103, 1790-1800. 
Satnoianu, R. A., Menzinger, M. \& Maini, P. K. [2000] "Turing instabilities in general system," J. Math. Biol. 41, 493-512.

Segel, L. A. \& Jackson, J. L. [1972] "Dissipative structure: An explanation and an ecological example," $J$. Theoret. Biol. 37, 345-559.

Seirin Lee, S., Gaffney, E. A. \& Monk, N. A. M. [2010] "The influence of gene expression time delays on Gierer-Meinhardt pattern formation systems," Bull. Math. Biol. 72, 2139-2160.

Shi, J.-P. \& Wang, X.-F. [2009] "On global bifurcation for quasilinear elliptic systems on bounded domains," J. Diff. Eqs. 246, 2788-2812.

Su, Y., Wei, J.-J. \& Shi, J.-P. [2009] "Hopf bifurcations in a reaction-diffusion population model with delay effect," J. Diff. Eqs. 247, 1156-1184.

Szili, L. \& Tóth, J. [1997] "On the origin of Turing instability," Z. Math. Chem. 22, 39-53.

Turing, A. M. [1952] "The chemical basis of morphogenesis," Phil. Trans. Roy. Soc. London B 237, 37-72.

Varea, C., Hernandez, D. \& Barrio, R. A. [2007] "Soliton behaviour in a bistable reaction-diffusion model," $J$. Math. Biol. 54, 797-813.

Wang, J.-F., Shi, J.-P. \& Wei, J.-J. [2011] "Dynamics and pattern formation in a diffusive predator-prey system with strong Allee effect in prey," J. Diff. Eqs. 251, 1276-1304.

Wijeratne, A. W., Su, Y. \& Wei, J.-J. [2009] "Hopf bifurcation analysis of diffusive Bass model with delay under 'negative-word-of-mouth'," Int. J. Bifurcation and Chaos 19, 1059-1067.

Xu, C. \& Wei, J.-J. [2012] "Hopf bifurcation analysis in a one-dimensional Schnakenberg reaction-diffusion model," Nonlin. Anal. Real World Appl. 13, 19611977.

Yan, X.-P. \& Li, W.-T. [2008] "Stability and Hopf bifurcation for a delayed cooperative system with diffusion effects," Int. J. Bifurcation and Chaos 18, 441-453.

Yan, X.-P. \& Li, W.-T. [2009] "Stability of bifurcated periodic solutions in a delayed competition system with diffusion effects," Int. J. Bifurcation and Chaos 19, 857-871.

Yi, F.-Q., Wei, J.-J. \& Shi, J.-P. [2009] "Bifurcation and spatiotemporal patterns in a homogeneous diffusive predator-prey system," J. Diff. Eqs. 246, 1944-1977. Yi, F.-Q., Liu, J.-J. \& Wei, J.-J. [2010] "Spatiotemporal pattern formation and multiple bifurcations in a diffusive bimolecular model," Nonlin. Anal. Real World Appl. 11, 3770-3781. 